\title{
Investir dans la Société canadienne des pharmaciens d'hôpitaux
}

\author{
Jody Ciufo
}

$\int \mathrm{e}$

e suis très touchée de l'accueil chaleureux que m’ont réservé les membres de la Société canadienne des pharmaciens d'hôpitaux (SCPH), nos administrateurs et les filiales de partout au pays, mais aussi notre équipe à Ottawa tout au long de ma première année à titre de directrice générale de la SCPH. Une telle entrée en matière est particulièrement importante pour moi qui suis une nouvelle venue dans les secteurs de la pharmacie et des soins de santé. Les pénuries de médicaments, les programmes d'assurancemédicaments, l'excellence en matière de pratique, la crise des opioïdes et les autres enjeux au cœur des soins offerts aux patients sont des réponses à certains des défis sociaux sur lesquels je me suis penchée tout au long de ma carrière au sein d'associations nationales sans but lucratif. Pouvoir travailler avec les professionnels de la pharmacie dont la préoccupation principale consiste à veiller au bien-être des Canadiens, en particulier des plus faibles et des plus vulnérables d'entre nous, est une source d'inspiration au quotidien quand on se trouve dans cette position.

Le conseil m'a confié un mandat clair visant à valoriser l'image des pharmaciens d'hôpitaux, à augmenter le nombre d'adhérents, à susciter l'engagement de la communauté des pharmaciens d'hôpitaux et à amplifier la voix collective de la SCPH. Mais surtout, les membres du conseil m'ont communiqué leur ferme engagement à apporter des changements fondamentaux à la Société et ils m’ont assurée de leur pleine collaboration pour qu'ils se concrétisent.

Ma prédécesseure, Myrella Roy, a affronté un défi d'envergure lorsqu'elle est devenue directrice générale. Grâce à son ingéniosité et à son travail acharné, la $\mathrm{D}^{\text {re }}$ Roy a bâti une société florissante au cours des 15 ans qu'elle a passés à la tête de la SCPH. Pendant cette même période, l'ensemble du monde associatif s'est heurté à des changements qui ont bouleversé l'adhésion aux sociétés dans le monde entier. La croissance des associations de niche, la concurrence accrue visant à obtenir davantage de fonds des commandites et des adhésions ainsi que les compressions importantes dans le cadre professionnel effectuées par les employeurs ont remis en question les adhésions durables aux sociétés dans presque tous les secteurs. Dans notre secteur, des changements importants au sein de l'industrie pharmaceutique ont affecté la nature du financement et des relations entre les industries, le secteur des soins de santé, les établissements d'enseignement et, bien sûr, les associations professionnelles. Les modèles commerciaux qui ont favorisé l'épanouissement de la SCPH ne suffisent plus pour offrir à nos membres les avantages qu'ils méritent et auxquels ils s'attendent.

Afin d'effectuer le changement fondamental nécessaire à l'évolution du nouveau monde associatif, le comité exécutif a travaillé d'arrache-pied avec le conseil, les présidents des filiales ainsi que les conseils affiliés pour créer une stratégie visant à retrouver une croissance durable. Les dernières années ont été le témoin du déclin du nombre d'adhésions et des revenus de diverses sources. Nous avons entrevu des années de déficit à long terme et avons conclu qu'un choix s'imposait : réduire notre offre de programmes et services pour nos membres afin d'équilibrer le budget ou alors définir un plan pluriannuel visant à investir dans la Société pour faire en sorte qu'elle soit encore présente pour soutenir l'avenir de la pratique de la pharmacie d'hôpital.

Nous avons évidemment choisi d'investir.

Dans un élan de soutien unanime, le Bureau national de la SCPH, nos filiales et conseils affiliés ont accepté de contribuer financièrement à l'atteinte de l'objectif commun, qui consiste à être plus axé sur les membres, mais aussi à offrir plus de programmes, de services, d'occasions de formation et de pertinence à la génération actuelle des membres, des sympathisants et des étudiants. Au cours des quatre années à venir, nous investirons ensemble près d'un million de dollars pour retrouver notre équilibre budgétaire d'ici 2023 - le Bureau national contribuera à hauteur de $85 \%$, les filiales à hauteur de $12 \%$ et les conseils affiliés à hauteur de $3 \%$.

Nous appelons cela un " co-investissement ", terme qui reflète l'aspect collaboratif de notre travail visant à visant à assurer la durabilité. Pendant quatre mois, le conseil, les filiales et les conseils affiliés se sont penchés sur plusieurs options. Ils ont convenu de ce plan en vertu duquel les filiales paieraient un montant selon une formule calculée en fonction du pourcentage 
d'adhérents à la SCPH et de leur capacité de payer. Nous avons tous convenu qu'il s'agissait là d'une manière équitable et abordable de procéder et que c'était ce qu'il y avait de mieux à faire.

La stratégie demandera un investissement important en infrastructure humaine et technologique pour que nous puissions fournir de plus grands avantages aux membres. Le Bureau national compte désormais un service de communications et de marketing dirigé par une directrice chevronnée, Clara Wicke. Il a pour mission de rapprocher les membres de notre communauté et de donner une voix à la profession bien au-delà de la seule adhésion. Le Bureau compte également une plus grande équipe de pratique en pharmacie, dirigée par Christina Adams, qui occupe le poste nouvellement créé d'agente principale en pharmacie. Elle s'associera à deux spécialistes de la pratique professionnelle pour élargir notre offre de programmes et de services et pour aider nos comités de bénévoles à atteindre leurs objectifs. La refonte de nos systèmes des technologies de l'information (T.I.) permettra à la SCPH de déterminer les besoins des divers segments du secteur de la pharmacie d'hôpital et d'y répondre.

Pour la première fois depuis 2013, la SCPH a mené une enquête nationale approfondie destinée à réunir des données sur la perception, les croyances et les préférences des membres. L’enquête a été conçue pour explorer les propositions développées pendant le travail portant sur la Stratégie de développement durable : les grandes idées en matière d'adhésion pour les techniciens en pharmacie, la spécialisation et le changement de nom de la Société ainsi que la proposition portant sur la valorisation de la SCPH et les priorités des membres. Nous avons mené simultanément une enquête distincte auprès des anciens membres pour comprendre les raisons de leur départ.

Près d'un membre sur trois a participé à l'enquête, qui prenait 20 minutes de leur temps. Il s'agit là d'un taux de réponse remarquablement élevé (1012 répondants sur nos 3602 membres). De plus, $9 \%$ ou 515 répondants sur les 5585 anciens membres ayant renoncé à leur adhésion au cours des 10 dernières années ont souhaité faire part de leur opinion.

Nous devions entendre directement de la part de nos membres les raisons pour lesquelles ils adhéraient à la SCPH. Les trois raisons principales étaient les suivantes : «Appartenir à une association professionnelle qui représente des normes de pratique élevées ", "Rester informé des nouvelles et des développements dans le domaine de la pharmacie d'hôpital " et "Accéder aux informations qui améliorent mon travail ". L'excellence professionnelle souligne notre raison d'être.
Il n'est donc pas surprenant d'apprendre qu'un nombre important de membres (86\%) se décrivent comme très satisfaits et plutôt satisfaits du Journal canadien de la pharmacie d'hôpital (JCPH) et le considèrent comme une ressource particulièrement précieuse. Seul le Programme de résidence a atteint un score plus élevé (avec un degré de satisfaction de 89 \%). De plus, le Journal est le produit ou le service de la SCPH que les membres connaissent le mieux, puisque seuls $4 \%$ disent ne pas le connaître. Les membres accordent une grande importance aux normes et à la qualité élevées sur lesquelles insistent le comité de rédaction et l'équipe de production.

Dans le cadre de sa Stratégie de développement durable, la SCPH s'est engagée à faire régulièrement le point sur le nombre d'adhérents, les revenus tirés des conférences, les statistiques relatives aux médias sociaux et les autres indicateurs clés de la performance. L'enquête nationale portant sur l'opinion des membres à l'égard des programmes, des services et de l'expérience générale d'adhésion, qui sera organisée chaque année, servira de mesure déterminante permettant d'évaluer les progrès et d'effectuer des ajustements en cours de route.

L'année dernière a permis d'ouvrir la voie à un nouveau départ de la SCPH, car le Plan stratégique de 2015-2020 touche à sa fin. La phase de planification pour l'année 2020 et les suivantes est en bonne voie, et cette année verra le lancement de notre nouveau plan stratégique de 2020-2023.

La Stratégie de développement durable constitue la promesse commune de notre communauté, qui se porte garante de l'évolution favorable de la pratique de la pharmacie hospitalière. Je vous encourage à vous réinvestir au sein de notre communauté dynamique en faisant du bénévolat à l'échelle de la filiale ou à l'échelle nationale, en participant à une conférence de la SCPH, en nous accordant une nouvelle chance si vous avez laissé expirer votre titre d'adhérent ou en réfléchissant avec un esprit critique aux thèmes abordés dans ce numéro du JCPH.

Je suis réellement honorée de faire partie de cette communauté et j'ai hâte de mettre à profit l'inspiration constamment nourrie par le leadership et l'expertise de nos bénévoles et des membres de notre personnelo.

[Traduction par l'éditeur]

Jody Ciufo, M. B. A., est directrice générale de la Société canadienne des pharmaciens d'hôpitaux. 